\title{
PERSPECTIVE ARCHITECTURE AND COMPONENTS OF COMPUTER NETWORKS
}

\author{
Yaroslav Nykolaychuk ${ }^{1)}$, Nazar Krutskevych ${ }^{1)}$, \\ Oleg Zastavniy ${ }^{1)}$, Taras Grinchyshyn ${ }^{2)}$
}

\author{
1) Ternopil Academy of National Economy, Lvivska Str. 11, nazar777@yahoo.com \\ 2) Ivano-Frankivsk National Technical University of Oil and Gas, Karpats'ka Str. 17
}

\begin{abstract}
This article is about using perspective architecture and components of computer networks will permit to increase productivity and reliability of the specialized computer networks not at the expense of escalating elements of system.
\end{abstract}

Keywords: - specialized computer systems, memory of multiple accesses, open optical channels, autonomous sensors

\section{INTRODUCTION}

Now compared with fast development as well as mass introduction of standard local computer networks and regional computer networks, specialized computer systems (SCS), problemoriented and low-level computer networks are becoming very important. Thus the essential factor of their efficiency is a choice of architecture and components of computer networks especially on a basis of high parallel architectures.

The development of networks determines the directions of study of objects of management as a source of the information, which are a subject of selection, transmitting, processing and storage in databases. For identification of statuses of structural elements of the computer networks and creation of characteristics of control, the important element is research of models of such objects.

\section{STAR-MAIN ARCHITECTURE OF NETWORKS}

The most universal, at construction of information--measuring and multiprocessor structures, is a star-main architecture (fig. 1) [1].

On the basis of multiple access (associative) memory such network can be used: in classical multiprocessor system; in a ring structure at examining a star as an element of information processing; in systems with regular structure and general trunk. The association in one block of the switchboard and memory cells has permitted practically without limits to increase quantity of modules, radially connected with the centre of each local area network of "star" type.

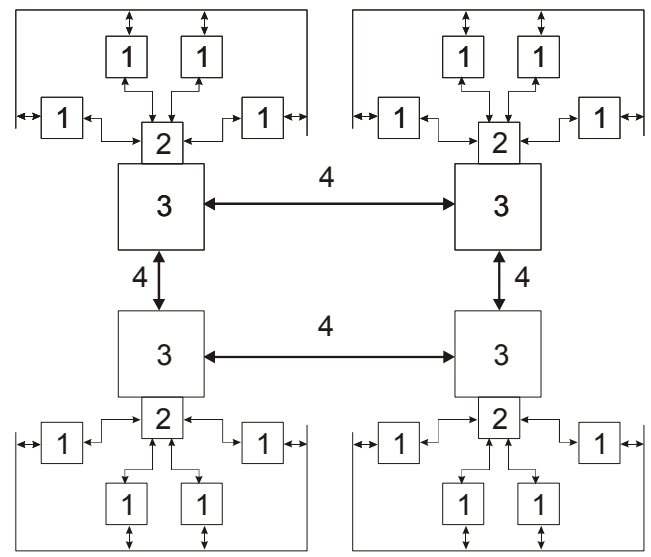

Fig. 1 - Star-main architecture (1- abonents, 2controllers of a network, 3- memories of multiple access (MMA), 4 - high-speed information data highway).

One of lacks of the majority of architectures, which limits their wide implementation, is use of network resources and capture of channels by lock devices.

Use of MMA as operative memory of multiple access with parallel reading - recording of the data, enables to realize star-main structure, in which the independent parallel work of channels in any of trunks and data exchange between trunks is carried out It permits practically unlimited number of the abonents of multilevel information networks communicate independently .

\section{MEMORY OF MULTIPLE ACCESSES}

The basic element of star-main architecture is 
memory of multiple access, which consists of the basic functional nodes: the decoder and switchboard of inputs; the switchboard of outputs; the circuits of management of recording / reading; the circuits of synchronization; "mail boxes" of random access memory (RAM) and read only memory (ROM) [2].

Memory of multiple accesses (MMA) is organized as set of "mail boxes". Each "mail box" represents a set of cells of memory, in which the package of the information is located. The given package also is that conditional individual information package, which is transferred from the abonents to MMA and from MMA to the abonents. The sizes of this package, the depth of "mail box" depends on the requirements of time restriction. All "mail boxes" are divided into "boxes", in which the information can be recorded and read out, and also "boxes" in which the data can only be read out. For any of the abonents, depending on the requirements of concrete network realization, some "mail boxes", in which the appropriate subscriber has the right to write down the information, can be allocated. Any of other abonents physically can't write down the block of the data in these boxes. Any subscriber can read a package from any "mail box". Therefore some "boxes" are used only for reading. Such "boxes" carry out ROM functions (service programs of the user) in which information does not change during long time [2].

The decoder and switchboard of inputs determine "mail box" number of any abonent. Besides the type of operation (reading / recording), for any of channels is determined. The switchboard of outputs connects the appropriate "mail boxes" to the channel, was necessary to establish communication (Fig. 2).

In a fig. 2 following designations are accepted: the program facilities - MB-RAM - mail box of the operative remembering device (recording / reading); the program facilities - MB-ROM - mail box of the constant remembering device (reading of the information).

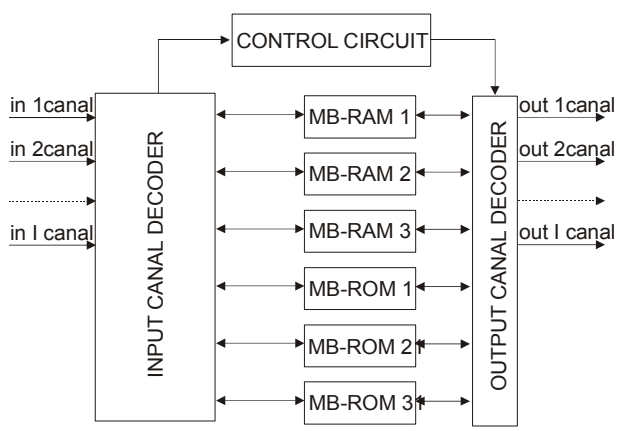

Fig. 2 - Structure of memory multiple access. 3.

The diagram of work MMA is submitted on a fig.

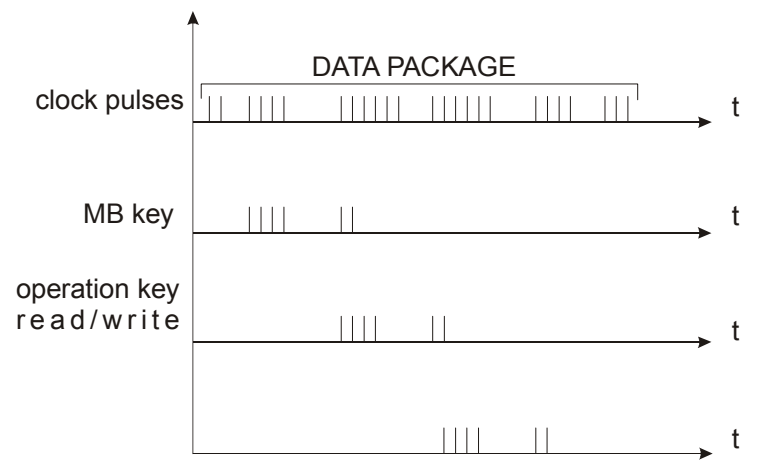

Fig. 3 - The diagram of work MMA.

The frequency of a synchronizing sequence corresponds to minimally possible time of reading / recording of element base, which applied in concrete cells of memory "mail boxes".

The absence at the certain moment of next clock pulse means the beginning of data package. With the beginning of dialogue acts " $n$ " bit, which is code of the "mail box" to be connected, then following " $k$ " bit, which determines a type of operation, and after that " $\mathrm{m}$ " bit sequence of an information part.

The work of the digital automatic device in a cyclic mode permits precisely to establish time of access to system resources [2].

$$
\text { Tdost }=\mathrm{tn}+\mathrm{tk}+\mathrm{tm},
$$

where $\mathrm{tn}+\mathrm{tk}$ - time of an address part, and tm time of information part to a date package.

The coding of an address part in codes of a Galois field has permitted considerably to simplify the circuit of the decoder of channels. It has resulted in development of transparent and regular structure of the switchboard of channels capable to be combined with the similar circuits. Thus the there appeared the possibility to increase a number of possible abonents, which work with general system resources. Increase of the abonents are limited only theoretically by length of " $n$ " bit key of number of the "mail box" of an address part to a package of the data. The hardware realization of the entrance decoder has permitted simultaneously in the current cycle of the digital automatic device to process the information from any quantity of the abonents wishing to establish communication with appropriate "mail boxes" and files.

\section{AUTONOMOUS SENSOR CONTROLS}

Using of devices of remote diagnostics of industrial objects is an integral compound of all automated management systems, which permits considerably to increase their efficiency. Nevertheless majority of these devices can't work in critical conditions (for example: at disconnect of a 
power or damage of communication lines).

The monitoring system of object operation consists of autonomous sensor controls (AS) [3]. AS placed on the object, which with the help of sensor controls define a status of an object, and central receiver, which is placed at the central station (CS). At normal work of object AS periodically transfers a code of serviceable work of object, which permits to supervise serviceability of AS and the object, and at occurrence of malfunctions begins to transfer a code of malfunction. The given system concerns to a class of systems with SSS. Due to specific features of these systems there is an opportunity to organize simplex communication AS with CS, and CS can accept the information with several AS simultaneously, that transfer the data in one strip of frequencies, and it permits to use one receiver for many AS and simplifies escalating and reduces cost of the system. Block scheme of the monitoring system on base AS represented on a fig. 4 .

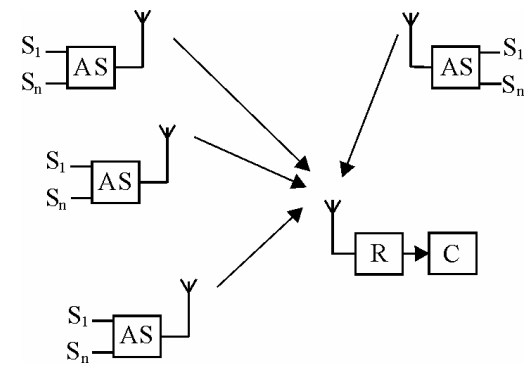

Fig. 4 - Block scheme of the monitoring system on base AS.

AS is a device of data collection and data transfer and contains the following functional sites: sensor controls, block of formation of correlation codes, the broadband transmitter, antenna and block of a uninterrupted power. The block diagram AS is represented on a fig. 5 .

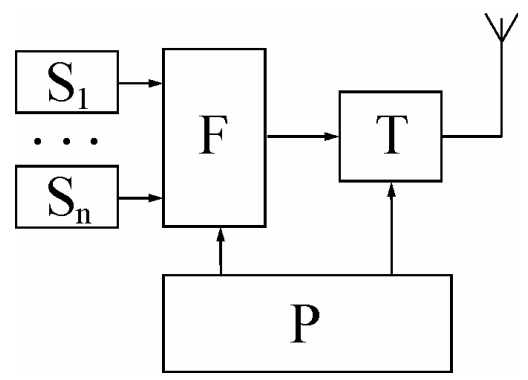

Fig. 5 - The block diagram AS.

Sensors $(\mathrm{S} 1, \ldots, \mathrm{Sn})$ are used for the equipment monitoring, , monitoring of the signal system sensors, power supply lines, integrity of information cables. The broadband transmitter $(\mathrm{T})$ with the directed antenna is used to transmit information on CS. In conditions of direct visibility the antenna can be replaced on optical emitter. On CS there is a receiver (Fig 5), which consists of broadband receiver (R) and computer (C) on which the processing of the accepted signal is carried out by realization of correlation between the accepted signal and reference codes.

There are many ways of distribution of a signal in a wide strip of frequencies. But for commercial use only technologies frequency hopping $(\mathrm{FH})$ and direct sequence (DS) are permitted. They are the most popular methods of reception SSS.

Determining in the theory of transfer spread spectrum signals is CS, or correlation codes. They define link speed of the information and degree of noise stability.

The best correlation properties are posses Barker codes [3] - signals with binary components, which have minimally possible at given $\mathrm{n}$ a level of lateral petals of correlation functions, which does not exceed $1 / \mathrm{n}$ of the basic. Such signals are found only for the certain values. The correlation function for Barker codes can be calculated according to the formula

$$
\phi_{(x, y)}(j)=\sum_{i=0}^{n} \operatorname{sign}\left(\dot{x}_{i}\right) \cdot \operatorname{sign}\left(\dot{y}_{i}\right)
$$

In which - Barker code, - three-stable compared signal, where,

$$
\operatorname{sign}\left(y_{i}\right)=\left\{\begin{array}{c}
1 ; y_{i}>0 \\
0 ; y_{i}=0 \\
-1 ; y_{i}<0
\end{array} .\right.
$$

Despite of good correlation properties the Barker codes have one essential lack, namely small length, which creates inconveniences at their use in channels with a high level of handicaps. Therefore to transmit the information on the large distances on channels of communication with a high level of handicaps $\mathrm{M}$ - sequences are frequently are applied [4], or the sequences of maximal length, which are formed on the basis of not reduced algebraic polynomials according to expression where - binary value of not reduced of algebraic polynomial, which forms a code recurrence key for $\mathrm{M}$ - sequence.

The majority of M-sequences have the low correlation characteristics, but the plenty of these codes permit to find the compromise between length of a code and its correlation properties.

Perspective there is use of two-dimensional signals. The example of synthesized twodimensional signal is represented in figure 6 . In the given signals the matrix of $\mathrm{m} / \mathrm{h}$ size is used. By transfer the given matrix is broken into lines and is consistently transferred on the channel of communication, on the receiver the accepted signal again develops in a matrix and the correlation 
according to the formula (1) is carried out. That is the correlation function for lines and columns of a matrix is calculated.

$$
K_{(x, y)}=\sum_{j=1}^{h} \sum_{i=1}^{m} x_{i, j} \cdot y_{i, j}+\sum_{i=m}^{1} \sum_{j=1}^{h} x_{i, j} \cdot y_{i, j}
$$

where $\mathrm{h}$ - height of a matrix, $\mathrm{m}$ - width of a matrix.

In a fig. 7 correlation function two-dimensional of a signal is represented. It is calculated by formula (1).

From the given figure it is visible, that the level of lateral petals is low concerning the basic peak, in this case makes $4 \%$. For comparison the level of a petal $\mathrm{M}$ - sequence, from which in this case twodimensional signal was synthesized, makes $8 \%$. The basic correlation peak of the given code is twice greater than in a M-sequence of similar length.

$\begin{array}{lllll}1 & 0 & 0 & 1 & 0 \\ 0 & 1 & 0 & 1 & 0 \\ 1 & 0 & 0 & 0 & 0 \\ 0 & 0 & 1 & 1 & 1 \\ 0 & 0 & 1 & 1 & 1\end{array}$

Fig. 6 - Two-dimensional correlation signal.

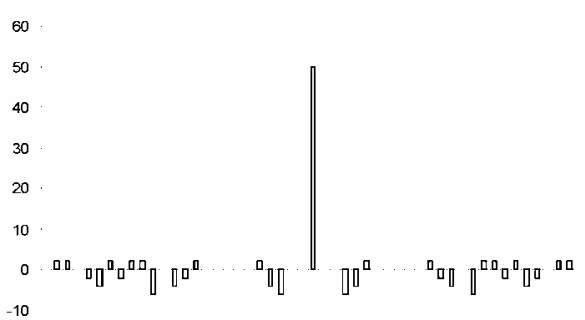

Fig 7 - Correlation function of two-dimensional signal.

The analysis of the characteristics CS shows, that the realization of sensor controls for protection of objects of control is expedient to carry out on a basis of two-dimensional signals. The stated principles can be effectively used at organization of wireless channel of communication on a SSS basis, as twodimensional signals at identical length in comparison with M-sequences have better correlation properties.

\section{THEORY AND PRINCIPLES OF CONSTRUCTION OF COMPONENTS OF COMPUTER SYSTEMS AND NETWORKS ON THE BASIS OF BASES RADEMAHER, CRESTONSON, GALOIS}

The basis Rademaher which is used as basic for the majority of computer facilities, settles the opportunities, which results in critical increase of word length of trunks, size of crystals in particular it is important for special processors.

The development of microelectronic base removes problems of inter-basic transformations. The theory of theoretical-numerical bases including those which derivate the systems of calculation: Rd (Rademaher) - binary; Cr (Crestonson) - residual classes; G (Galois) - system of calculation Galois.

Therefore urgent problem is development of new principles of construction of specialised processors with simultaneous use of several bases with orientation to vertical - information technology.

The different operations demand different speed, expenses of equipment, number of micro commands in different bases. For example in basis Rd the multiplication on 2 is realized by shift on one bit, and operation of increment through by digit shift. In that time the similar operation in basis Galois is realized by shift on 1 bit. The operation of multiplication in basis Crestonson is carried out by a matrix method for 1 step.

The following block diagram of three basses specialized processor RCG (fig. 8) is offered.

Orientation to vertical - information technology makes the trunks of address, of the data and control bit oriented, that permits considerably to reduce the sizes of the trunk and the crystal. And use of fast computing operations of different bases will result in reduction of number of machine steps and increase of productivity.

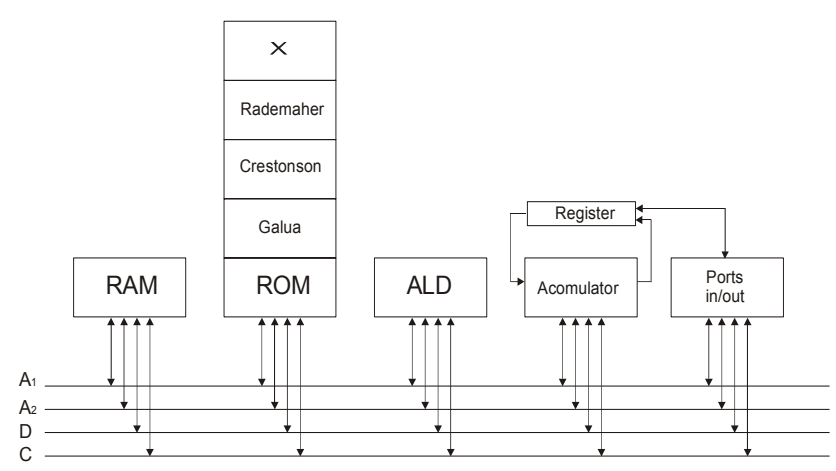

Fig. 8- the block Diagram of the RCG specialized processor.

\section{OPEN OPTICAL CHANNELS OF COMMUNICATION}

At organization of communication between objects in the specialized computer systems is perspective to use open optical channels (OOCC), which have the following advantages:

- high secretiveness of communication;

- development of a not used site of a spectrum of electromagnetic radiations;

- it is unnecessary the permits and payments on operation of optical system of communication;

- high bandwidth of the channel of 
communication;

- small dimensions of transmitting and acceptance modules, which are used for communication on distance up to $3 \mathrm{~km}$;

- security from electromagnetic handicap's.

The lacks of optical communication lines are the following:

- necessary high accuracy of prompting of antennas of the transmitter and receiver;

- influence of the characteristics of an atmosphere on reliability of communication.

- low efficiency of optical emitters.

On the basis of open optical channels of communication it is possible to realize different types of channels of communication: half-duplex, duplex, sinning, as well as such types of architecture of computer networks as star, ring, systolic and other.

Correlations between energy of transmitter and receiver signals is describe by equation:

$$
\begin{aligned}
& \left(P_{R}\right)_{\max }=\left(\frac{S_{1} \cdot S_{2}}{r^{2} \cdot \lambda^{2}}\right) \cdot \tau \cdot P_{A}, \\
& \text { or } \quad\left(P_{R}\right)_{\max }=\left(\frac{\pi^{2} \cdot d_{A}^{2} \cdot d_{R}^{2}}{16 \cdot R^{2} \cdot \lambda^{2}}\right) \cdot \tau \cdot P_{A}
\end{aligned}
$$

Where S1, S2 area of transmitter and receiver antennas, $\lambda$ - distance of frequency, $\tau$ - coefficient that consider light absorption in the atmosphere, $\mathrm{R}-$ distance without transmitter and receiver, $\mathrm{P}_{\mathrm{A}^{-}}$power of generator, $d_{A}, d_{R}$ - diameter of transmitter and receiver aperture.

The distance of communications system is calculated by equation (2) where different light absorption coefficients are used.

On the Fig.9 graphics of communication distance that was calculated using following parameters where $\mathrm{d}_{\mathrm{A}}=50 \mathrm{MM}, \mathrm{d}_{\mathrm{R}}=50 \mathrm{MM}, \mathrm{P}_{\mathrm{A}}=0.4 \mathrm{BT}, \tau=0 \ldots 1$. is presented.

From the graphic we can see that maximum communication distance of safe data receiving is $8 \mathrm{~km}$.

In the given time OOCC are widely used in computer networks. But it is also perspective use of the specified channels of communication for data gathering from sensor controls of technological parameters of the automated computer systems.

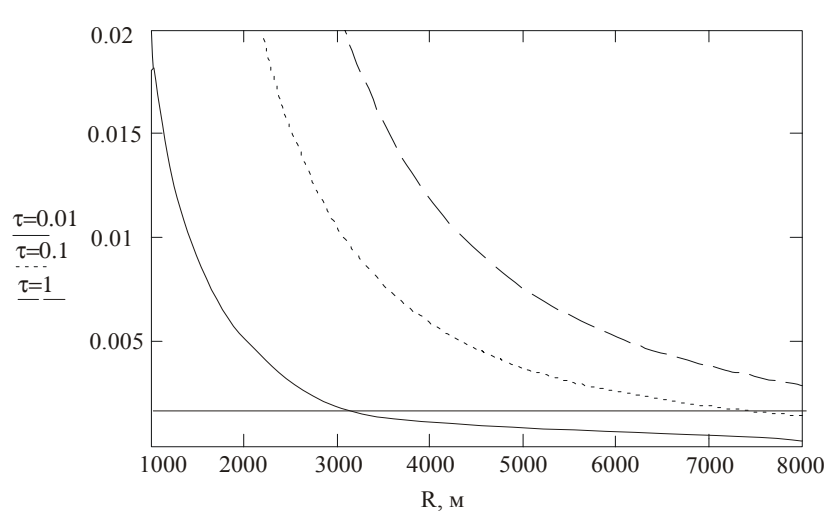

Fig 9 - Communication distance of open optical channel.

\section{CONCLUSION}

Use of proposed perspective architectures and components of computer networks will permit to increase the productivity, reliability, and decrease the cost of specialized computer networks, which are widely implemented on various enterprises, institutions and organizations.

\section{REFERENCES}

[1]Y. Nykolaychuk, N. Krutskevych. Prospects of use of starr-main architecture with memory of collective access in computer networks with deep paralleling. Measuring and computer facilities in technological processes, Khmel'nyc'k, 2002, №9, T2. an item 122-126.

[2]N. Krutskevych, Y. Nykolaychuk. Structure and functions of memory collective access based on Galois codes. Measuring and computer facilities in technological processes, Khmel'nyc'k, 2002, №9, T2. an item 126-129.

[3]Y. Nykolaychuk, O. Zastavniy. Methodology of construction of autonomous sensor controls for the distributed computer networks. The BULLETIN of Technological university Podillya, Khmel'nyc'k, 2002, №3, T1. an item 142-146.

[4]Varakin L. E. The theory of complex signals. Soviet radio, 1970. $-364 p$.

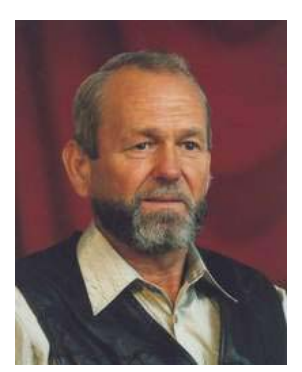

Yaroslav Nykolaychuk has graduated Lviv polytechnics institute in 1967 by speciality 05.13.05 "Elements and devices of computing equipment and control systems". He is a professor of Special Computer Systems Department at Institute of Computer Information Technologies of Ternopil Academy of National Economy, academician of Ukrainian Academy of Sciences of National Progress, deputy director for science affairs at Institute of Computer Information Technologies, PhD 
thesis's supervisor by specialities 05.13 .05 and 05.13.13.

Research areas: theoretical and numerical transformations; signal encoding and numerical processing in Galois base.

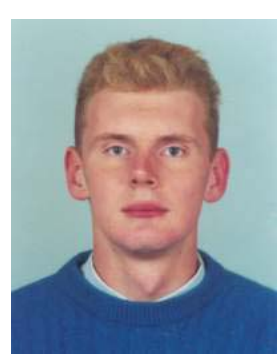

Nazar Krytskevych graduated Ternopil Academy of National Economy in 2002 with speciality "Computer Systems and Networks". At the moment he is PhD student at the department of Automatization Control at IvanoFrankivsk National Technical Univercity of Oil and Gas.

Areas of scientific interests includes: Autonomous Sensors, Distributed Networks, Robotics, Methods of parallel Processing and Accessing of Data, Bionics.
Oleh Zastavniy graduated Ternopil Academy of National Economy in 2002 with speciality "Computer Systems and Networks". At the moment he is PhD student at the department of specialized Computer Networks at Ternopil Academy of National Economy.

Areas of scientific interests includes: Autonomous Sensors,

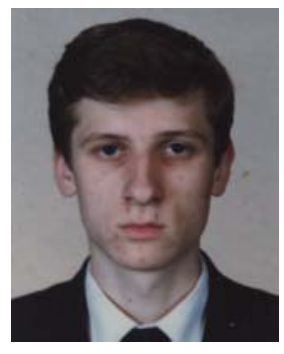
Distributed Networks, Robotics, Methods of Signal Processing, Bionics. 